\title{
Information modeling of the construction and demolition waste system
}

\author{
Alexander Ginzburg* \\ Moscow State University of Civil Engineering, Yaroslavskoe shosse, 26, Moscow, 129337, Russia
}

\begin{abstract}
Throughout the world, the intensive development of production is accompanied by a steady increase in waste generation. Ecological problems, problems of utilization and recycling of waste come to the fore. The effective functioning of the construction complex is largely determined by the effective organization of the construction and demolition waste (CDW) circulation. Considering the scale of construction, forecasting the volume of waste formation in order to effectively organize the system of economic turnover of CDW is a very relevant scientific and practical problem. Management of CDW flows in Smart cities should be based on information models containing the necessary data on the types, forms and volumes of waste. The paper presents such an information model and a mathematical method of predicting the formation of the CDW. Information modeling of the composition and volume of CDW formation allows on the basis of the developed mathematical apparatus to provide an analysis of construction development trends. It allows to carry out construction production operational and strategic planning and management in terms of the CDW formation, processing and disposal. Analysis of the construction, reconstruction and demolition CDW volume dynamics information model is the basis for further design of treatment plants future scheme.
\end{abstract}

\section{Introduction}

Throughout the world, the intensive development of production is accompanied by a steady increase in waste generation. Environmental problems, waste disposal and recycling problems come to the fore ([1-4]). The construction complex effective functioning in modern economic conditions is largely determined by the system of construction and demolition waste (CDW) economic turnover effective organization ([5-7]).

The organization of CDW recycling and utilization is a long and expensive process. Preparation of the necessary landfills and treatment facilities requires a significant time and money investment. It is necessary to plan in advance the order of waste disposal, the required treatment capacity and equipment ([8]). Given the scale of construction, increasing requirements to ensure environmental safety, forecasting of waste generation volume for the purpose of CDW economic turnover effective organization is a very urgent scientific and practical problem ([1-4]).

\footnotetext{
* Corresponding author: ginav@mgsu.ru
} 
In Smart cities, the issues of regulation of receipt, movement, disposal and treatment of CDW can be solved on the basis of construction and demolition waste using information model, i.e. obtaining information on the composition, objects of formation, volumes of processing and disposal of waste on the basis of technological regulations included in the project documentation ([9-15]).

The process of construction and demolition waste management for each specific object of waste formation is determined by the special CDW handling technological regulations.

All waste producers, regardless of the form of ownership and departmental affiliation, are required to have technical and technological documentation regulating the process of handling the construction and demolition waste generated by them in the form of technological regulations. It should reflect complete and accurate information on the whole process of waste management and demolition from the moment of their formation to the moment of their use or disposal ([16-21]).

Technological regulations for under construction, repaired, reconstructed, dismantled, demolished buildings, structures and utilities or industrial facilities are developed separately for each CDW formation object at the stage of project documentation preparation.

This should be provided:

- sanitary-ecological and radiation inspection of structures and materials subject to processing and further use;

- order of the buildings and constructions demolition organization and action for environmental protection for the period of demolition and burial of the polluted waste.

Waste producers are obliged to carry out separate collection (sorting) and temporary storage of construction and demolition waste to be processed and further used, according to the set of items having a single direction of use, as well as separate collection and temporary storage of construction and demolition waste to be buried of according to hazard classes.

On the basis of the General information flow of data on the treatment of CDW, the need to develop a program for the creation of new production facilities for the processing of $\mathrm{CDW}$, the introduction of new methods for the removal of construction waste, and, as a consequence, the suppression of the practice of illegal placement of waste in unauthorized landfills and the increase in the volume and types of recycled construction waste should be determined.

The organization of construction, reconstruction and demolition volumes forecasting, CDW formation volumes forecasting are an integral part of complex actions for ensuring rational functioning of all economic CDW turnover system.

\section{Methods}

As part of the study revealed that construction waste is formed mainly in the performance of the following types of work:

- buildings and structures reconstruction;

- buildings and structures repair;

- building materials, parts and structures production;

- buildings and structures demolition;

- new construction.

The analysis shows that the contribution of different directions in the scheme of CDW regional turnover not equivalent.

Based on the features listed construction programs, for the combined analytical evaluation of characteristics of construction complex activity with the purpose of studying schemes of regional economic CDW turnover, enlarged can distinguish 3 types of construction: 
- new construction;

- reconstruction (including overhaul);

- demolition (including dismantling).

It is these three areas of construction activity that determine the composition and volume of the formed CDW in significantly different ways.

A significant impact on the waste generation system in construction has the type of object. Depending on the type of constructed, reconstructed or demolished object is formed not only a different volume of $\mathrm{CDW}$, but also differently organized scheme of their disposal and processing.

In order to unify the huge variety of construction projects, it is advisable to identify a number of groups of objects with the following characteristics for the analysis:

- objects included in the selected group provide the same type of CDW structure for this type of construction;

- - the total volume of CDW formation for the objects of the selected group makes a significant contribution to the total mass of the CDW for the considered territorial formation.

Thus, based on the specifics of the regional scheme of economic CDW turnover, the main directions for analysis by types of construction and facilities should be identified.

The proposed method of forming the information base of the CDW is based on statistical data on the CDW volume for various types of construction and demolition. Analysis and processing of these data allowed us to formulate the following principles, which form the basis for the formation of the regulatory framework of the CDW:

- norms of waste formation are formed for each type of waste by types of objects and types of construction or demolition;

- norms of waste formation determine the volume of this type of waste per unit capacity of the object of this type for this type of construction or demolition;

- the volume of this type of waste per unit capacity of the object is a random variable, because its value is influenced by numerous additional factors: the conditions of construction (or operation of demolished) buildings, their age, etc., but the influence of these factors is not decisive.

For this type of object, this type of construction and demolition, the random value of the CDW volume per unit of the object capacity has a fixed normal distribution that does not change over a 5-10-year period of time. This fact is explained by the lack of significant changes in the technology of building materials production and construction works for a relatively short period of time.

As a norm, the average value of the available statistical sample of waste values per unit capacity of the object for this type of construction or demolition is taken.

When solving a number of tasks, for example, planning the construction of enterprises for the processing of CDW or tasks related to long-term (for a period of 10-15 years) planning, instead of a point estimate (norm) of CDW, it is advisable to use an interval estimate, i.e., instead of a fixed value of the norm, set the interval of its possible values.

Let us consider a mathematical model for assessing the composition and volume (formation of the regulatory framework) of the CDW for various objects and types of construction and demolition.

There are $I$ different types of objects, grouped by types of construction and demolition, numbered with indexes $i=1,2, \ldots, I$, and $J$ CDW types numbered with indexes $j=1,2, \ldots, J$.

There are statistics on the composition and volume of CDW by type of objects and types of construction over the past few years. Namely, for each object and each type of construction, there is a sample of capacity values $U_{i}^{s}$, constructed (or demolished) objects 
of the $i$-th type and the corresponding volumes of CDW $V_{i j}^{s}, i=1,2, \ldots, I ; j=1,2, \ldots, J$; $s=1,2, \ldots, S_{i}$, where $S_{i}$ is the sample size for the given values of $i$.

The analysis of the available statistical data allowed to assume that the random value is the ratio of the $\mathrm{CDW}$ volume to the capacity of the construction object, i.e. the rate of waste formation

$$
Q_{i j}=V_{i j} / U_{i}
$$

for fixed values of indexes $i$ and $j$ it has a normal distribution with time-independent parameters.

The hypothesis of the normal distribution of the random variable $Q_{i j}$ is verified by Pearson's chi-squared test. The test confirms the validity of the hypothesis.

The average values $q_{i j}$ of random values $Q_{i j}$, calculated for each pair of indexes $i=1,2 \ldots$ $I, j=1,2, \ldots, J$ by formulas:

$$
q_{i j}=\frac{\sum_{s=1}^{S_{i}} Q_{i j}^{s}}{S_{i}}
$$

As already noted, when planning the CDW volume indicators, in some cases, it is advisable to use not a point estimate - the CDW norm, but an interval - the minimum and maximum CDW value per unit of capacity for a given reliability indicator. In such cases, it is recommended to use interval estimates instead of the norms defined by formulas (2).

For each pair of indexes $i=1,2 \ldots I, j=1,2, \ldots, J$ estimates of the variance of $D_{i j}$ and the standard deviation $d_{i j}$ of the random variable $Q_{i j}$ are calculated from the formulas:

$$
\begin{gathered}
D_{i j}=\frac{\sum_{s=1}^{S_{i}}\left(Q_{i j}^{s}-q_{i j}\right)^{2}}{S_{i}-1} \\
d_{i j}=\sqrt{D_{i j}}
\end{gathered}
$$

As interval estimates of the norm of waste formation encouraged to take confidence intervals for the values of $Q_{i j}$ with reliability $\alpha=90 \%$ or $\alpha=95 \%$, determined by the formula:

$$
\left(q_{i j}-\frac{t_{\alpha, n-1} d_{i j}}{\sqrt{n}} ; q_{i j}+\frac{t_{\alpha, n-1} d_{i j}}{\sqrt{n}}\right)
$$

where

$t_{\alpha^{n-1}}$ - the value of the argument of the Student's $t$-distribution function corresponding to reliability $\alpha$,

$n=S_{i}$ - sample size in the $q_{i j}$ calculation.

As already mentioned, the analysis of statistical data on the composition and volume of the CDW $V_{i j}^{s}$ on the types of objects and types of construction, and capacity of objects $U_{i}^{s}$ over the past few years was put forward the hypothesis of normality of the distribution of the random variable $Q_{i j}=V_{i j} / U_{i}$ - volume of this waste per unit of the object capasity, where $i=1,2 \ldots I, j=1,2, \ldots, J$. Pearson's chi-squared test is used to confirm this conjecture.

Application of Pearson's chi-squared criterion involves splitting the whole set of available values of $Q_{i j}$ into several intervals. These values correspond to empirical (found from the available statistical data) and theoretical (calculated under the assumption of validity of the tested hypothesis) frequencies: $n_{1, n}, \ldots, n_{s}$ and $n_{1}^{\prime}, n_{2}^{\prime}, \ldots, n_{s}^{\prime}$ respectively. In the case of validity of the proposed hypothesis, the random variable 


$$
\chi_{\text {obsr }}^{2}=\sum_{i=1}^{s} \frac{\left(n_{i}-n_{i}^{\prime}\right)^{2}}{n_{i}^{\prime}}
$$

has a distribution $\chi^{2}$ with $p=s-3$ degrees of freedom.

Since the one-sided criterion rejects the tested hypothesis more "strictly" than the twosided one, the critical area is chosen on the basis of the requirement that the probability of the criterion falling into this area, assuming the validity of the hypothesis, is equal to the accepted level of significance $\alpha=0,05$.

Thus, the area of acceptance of the hypothesis is determined by inequality

$$
\chi_{\text {obsr }}^{2}<\chi_{c r i t}^{2}(\alpha ; r)
$$

where

$\chi_{c r i t}^{2}(\alpha ; r)$ - the value of the $\chi^{2}$ distribution argument, corresponding to the significance level $\alpha$ with $p=s-3$ degrees of freedom.

Estimates of the mean and variance of the random variable $Q_{i j}$ are given by formulas (2)-(4).

The mathematical model of CDW volumes forecasting is based on the developed mathematical model of CDW normative base formation. It comes from the known statistical data on the actual capacity of the objects built over the past few years. In case of insufficiency or inaccuracy of the planned indicators on the capacity of the facilities constructed for the planning period developed methods of forecasting of these indicators.

The forecast of construction volumes is made on the basis of statistical data on the construction volumes available over the past few years.

Consider the mathematical model of predicting the capacity of construction and demolition and the corresponding CDW volumes.

There are statistics for the last few years on the capacity of the constructed objects $U_{i k t}$, grouped by types of objects and types of construction, the location of the object, and years, where $i=1,2, \ldots, I, k=1,2, \ldots, K, t=1,2, \ldots, T$.

Index $i$ indicates the type of object and type of construction.

Index $t$ corresponds to the year, for convenience, a relative time scale is selected: the value $t=1$ corresponds to the first year, for which there is data on the actual capacity of the constructed objects, and the value $T$ - the last.

Index $k$ corresponds to the conditional number of the area in which the object is built, it is called the index of the location of the object. The need for the introduction of the object location index is due to the specifics of a number of planning tasks, in particular, the task of placing enterprises for processing CDW.

It is required to estimate the corresponding capacity for $t=T+1, T+2, \ldots, T+r$, where $r$ is the prediction period.

The paper proposes and implements the following procedure for assessing the capacity of construction and the corresponding volumes of CDW:

1. Analysis of the available data on the capacity of the facilities built over the past few years and the choice of the type of dependence characterizing the changes in construction capacity by year.

2. Estimation of dependence parameters of construction capacities change on years.

3. Estimation of the error between the calculated and actual values of the construction capacity.

4. Forecasting of construction capacity for the planned period.

5. Forecasting of CDW volumes for the planned period. 
The choice of the type of the dependence characterizing changes of construction capacities by years is made on the basis of the analysis of statistical data on the actual capacities of the constructed objects.

The values of the parameters of the selected dependencies are determined by the ordinary least squares method, i.e. the values of these parameters are calculated from the condition:

where

$$
\Phi(a, b, c)=\sum_{t=1}^{T}\left(F_{i k}(a, b, c, t)-U_{i k t}\right)^{2} \rightarrow \min ,
$$

$a, b, c$-parameters of the function $F_{i k}(a, b, c, t)$, approximating the values of $U_{i k t}$, at fixed values of indexes $i$ and $j$ for all $t=1,2, \ldots, T$.

The standard deviation of the actual volume value from the calculated value is used as an error estimate:

$$
\delta=\sqrt{\frac{\Phi(\mathrm{a}, \mathrm{b}, \mathrm{c})}{\mathrm{T}}}
$$

Thus, to obtain the value of the predicted indicator of all dependencies selected curve $F_{i k}(t)$ with the lowest value of the function $\Phi(a, b, c)$. The predicted values of the construction capacity are determined by the formulas:

where

$$
U_{i k t}=F_{i k}(t)
$$

$i, k$ - indexes of object type and its location,

$t=T+1, t=T+2, \ldots, t=T+r$ is the predicted period.

Having planned or calculated according to the formulas (10) values of the capacity building $U_{i k t}$ in the $t$-th year and formed the normative framework of CDW, it is possible to estimate the volume of CDW types. Namely, the volume of the $j$-th type of fixed asset for the $i$-th type of construction object in the $k$-th region is determined by the formulas:

where

$$
Q_{i j k t}=q_{i j} U_{i k t}
$$

$q_{i j}$ - value of the waste rate calculated by formulas (2).

The total volume of CDW for plan types in $t$-th year is calculated according to the formulas:

$$
Q_{j k t}=\sum_{i=1}^{I} Q_{i j k t}
$$

Substitution in formulas (11) instead of estimating the value of $q_{i j}$, estimates of its smallest and largest values $q_{i j}-\frac{t_{\alpha, n-1} d_{i j}}{\sqrt{n}}$ and $q_{i j}+\frac{t_{\alpha, n-1} d_{i j}}{\sqrt{n}}$, found by formulas (5), gives, respectively, the lower $Q_{i j k t}^{\min }$ and upper $Q_{i j k t}^{\max }$ values of the composition and volume of the $\mathrm{CDW}$, i.e. - interval estimate of the $q_{i j}$ value, with a given reliability.

Formed regulatory CDW database allows, in particular, to plan waste volumes by type. A prerequisite for this is the availability of planned indicators of construction capacity by types of facilities and types of construction at the regional level. Such indicators are available at all planning levels. However, as practice shows, these figures even for the next year are very conditional. This circumstance compels us to look for opportunities to predict the construction capacity for the planned period, based on the available data on the relevant indicators over the past few years.

For this purpose, we have analyzed the available data on the types of facilities and types of construction for the Moscow construction complex over the past few years. According to 
the results of the study, the work proposed the following three types of functional dependence of the capacity of the erected objects on time.

1. The calculation of the finite differences of the first and second order:

and

$$
\Delta U_{i k t}=U_{i k t}+1-U_{i k t}
$$

$$
\Delta_{2} U_{i k t}=\Delta U_{i k t}+1-\Delta U_{i k t}
$$

shows that for a number of objects and types of construction, the second differences of capacity (in some cases, the first differences) are almost constant. In these cases, the available data are approximated by polynomials of the second order, that is, by polynomials of the form $F_{i k}(t)=a+b t+c t^{2}$ (in particular, with $c=0$, straight lines $F_{i k}(t)=a+b t$ ). Dependencies of this type characterize uniformly accelerated (equally slow, in particular, uniform) processes, i.e. processes occurring in time with a constant positive, negative or zero growth rate (increment).

2. In some cases, (for example, for residential buildings of serial projects) graphics of the total capacity of the constructed (demolished) objects calculated on an accrual basis by year

$$
S_{i k t}=\sum_{p=1}^{t} U_{i k p}
$$

have the form of $S$-shaped curve.

In these cases, the total construction volumes $S_{i k t}$ in the paper are approximated by $S$ shaped Gompertz curves:

$$
F_{i k}(t)=a e^{b e^{c t}}
$$

The Gompertz functions were used in a number of studies to describe some growth processes with a value limited on the entire time interval. In particular, the capacity of residential buildings of the demolished series is the value limited by the total capacity of the existing buildings of this series.

3. The first difference of objects capacities logarithms and types of construction vary insignificantly over time. To approximate the capacity of such types of objects, a exponentiation dependence of the form $F_{i k}(t)=b t^{c}$ and, in a more general case, the dependence of the form $F_{i k}(t)=a+b t^{c}$ was proposed.

In determining the optimal values of the parameters $a, b, c$ included in the equations of the above functional dependencies, the least squares method was used, minimizing the value of function (8).

In the case of a second-order polynomial $F_{i k}(t)=a+b t+c t^{2}$, the exact optimal values of the desired parameters are found as follows.

In accordance with formula (8), the optimal values of the parameters $a, b, c$ are solutions of the system of equations:

$$
\left\{\begin{array}{l}
\frac{\partial F_{i k}(a, b, c)}{\partial a}=0 \\
\frac{\partial F_{i k}(a, b, c)}{\partial b}=0 \\
\frac{\partial F_{i k}(a, b, c)}{\partial c}=0 .
\end{array}\right.
$$

The partial derivatives of the left-hand sides of equations (17) are equal to:

$$
\frac{\partial F_{i k}(a, b, c)}{\partial a}=2\left(a T+b \sum_{t=1}^{T} t+c \sum_{t=1}^{T} t^{2}-\sum_{t=1}^{T} U_{i t}\right)
$$




$$
\begin{aligned}
& \frac{\partial F_{i k}(a, b, c)}{\partial b}=2\left(a \sum_{t=1}^{T} t+b \sum_{t=1}^{T} t^{2}+c \sum_{t=1}^{T} t^{3}-\sum_{t=1}^{T} t U_{i t}\right) \\
& \frac{\partial F_{i k}(a, b, c)}{\partial c}=2\left(a \sum_{t=1}^{T} t^{2}+b \sum_{t=1}^{T} t^{3}+c \sum_{t=1}^{T} t^{4}-\sum_{t=1}^{T} t^{2} U_{i t}\right)
\end{aligned}
$$

Thus, system (17) is linear with respect to the variables $a, b, c$ with the system of normal equations:

$$
\left\{\begin{array}{l}
a T+b \sum_{t=1}^{T} t+c \sum_{t=1}^{T} t^{2}=\sum_{t=1}^{T} U_{i t}, \\
a \sum_{t=1}^{T} t+b \sum_{t=1}^{T} t^{2}+c \sum_{t=1}^{T} t^{3}=\sum_{t=1}^{T} t U_{i t}, \\
a \sum_{t=1}^{T} t^{2}+b \sum_{t=1}^{T} t^{3}+c \sum_{t=1}^{T} t^{4}=\sum_{t=1}^{T} t^{2} U_{i t} .
\end{array}\right.
$$

The solutions of this system are the values of the parameters $a, b, c$ minimizing the functions (8).

In the approximation of the available data on the capacities of the objects under construction, not only the parameters of the parameters $a, b, c$, but also the methods of unconditional optimization that minimize the values (8) are used.

The approximation error is estimated by the formula (9).

The value of the predicted indicator $U_{i t}$ for the planned period is calculated by the formula (10).

\section{Conclusions}

The paper developed an approach to the formation of the construction and demolition waste formation system information model.

The calculations carried out for each type of object and type of construction for all types of waste confirmed the validity of the formulated hypothesis about the normality of the distribution of the random value of waste $Q_{i j}$. Norms of waste formation, calculated for all types of objects and types of construction for all types of waste, constitute the regulatory framework of the CDW.

The paper offered the mathematical model of construction capacities forecasting for the planned time period. Three types of functional dependence of the constructed objects capacity on time are revealed: polynomials of the second order (binomials), the Gompertz function, the exponentiation function.

The paper offered the mathematical model of CDW volumes forecasting for the planned time period, proceeding from norm of waste formation and the construction capacity forecast.

This work was financially supported by the Ministry of Science and Higher Education (state task \# 7.6932.2017/8.9).

\section{References}

1. V. Tam Rate of reusable and recyclable waste in construction / The Open Waste Management Journal, 4, pp. 28-32 (2011) 
2. J. Cheng, L. Ma A BIM-based system for demolition and renovation waste estimation and planning / Waste Management, Volume 33, Issue 6, -pp 1539-1551 (2013)

3. H. Yuan, A. Chini, Y. Lu, L. Shen A dynamic model for assessing the effects of management strategies on the reduction of construction and demolition waste / Waste Management, Volume 32, Issue 3, -pp 521-531 (2012)

4. H. Yuan, L. Shen Trend of the research on construction and demolition waste management / Waste Management, Volume 31, -pp 670-679 (2011)

5. A. Ginzburg, S. Kachanov Methodology for building automated systems for monitoring engineering (load-bearing) structures, and natural hazards to ensure comprehensive safety of buildings and constructions / International Journal of Applied Engineering Research ISSN 0973-4562 Volume 11, Number 3, -pp 1660-1665, (2016)

6. A. Ginzburg Sustainable Building Life Cycle Design / 15th International Conference on Topical Problems of Architecture, Civil Engineering, Energy Efficiency and Ecology, TPACEE-2016; Tyumen State University of Architecture and Civil Engineering: MATEC Web of Conferences, Volume 73, 02018 (2016)

7. A. Ginzburg LE IM: Living Environment Information Modelling / International Scientific Conference Environmental Science for Construction Industry, ESCI 2018; Ho Chi Minh City; Viet Nam: MATEC Web of Conferences, Volume 193, 05030 (2018)

8. V. Kulikov, P. Kagan, L. Sukneva Staging, formalization and typing of project procedures and processes of the industrial production / Applied Mechanics and Materials Vols. 405-408 -p 3343 (2013)

9. A. Volkov, V. Chulkov, R. Kazaryan, R. Gazaryan Cycle reorganization as model of dynamics change and development norm in every living and artificial beings / Applied Mechanics and Materials Vols. 584-586 -p 2685 (2014)

10. A. Volkov General information models of intelligent building control systems: basic concepts, determination and the reasoning / Applied Mechanics and Materials Vols. 838-841 -p 2973 (2014)

11. A. Volkov, Intelligence of buildings: formula, Promyshlennoe I grazhdanskoe stroitelstvo [Industrial and Civil Engineering], 3, -p 54 (2012)

12. A. Volkov, A. Sedov, P. Chelyshkov, Usage of building information modelling for evaluation of energy efficiency / Applied Mechanics and Materials Vols. 409-410 -p $630(2013)$

13. V. Garyaeva, N. Garyaev Integrated assessment of the technical condition of the housing projects on the basis of computer technology / Computing in Civil and Building Engineering Proceedings International Conference -p 1336 (2014)

14. A. Volkov, V. Chulkov, R. Kazaryan, M. Fachratov, O. Kyzina Possibility quantitative appraise components and guidance for constructional rearrangement of buildings attached to their confrontation, Advanced Materials Research, Vols. 1065-1069 -pp 2585-2588 (2015)

15. O. Kuzina Components of functional information model of city environment reorganization in interactive mode / MATEC Web of Conferences 73 -p 07013 (2016)

16. A. Ginzburg, S. Kachanov Technology for Enhancing Safety of Buildings and Constructions / International Journal of Applied Engineering Research ISSN 09734562 Volume 10, Number 20, -pp 40869-40872 (2015)

17. E. Gumerova, O. Gamayunova, L. Shilova The optimal decision of insulation in cladding structures for energy efficient buildings / MATEC Web of Conferences, $106-\mathrm{p}$ 06020 (2017)

18. H. Bargstädt, A. Nasir, E. Ignatova Can BIM support better working conditions for lowskilled labor? / 14th International Conference on construction applications of virtual reality CONVR, Published at Teesside University, UK, by Teesside University -pp 44$51(2014)$ 
19. E. Ignatova, H. Kirschke, A. Tauscher, K. Smarsly Parametric geometric modeling in construction planning using industry foundation classes / The 20th International Conference On The Applications Of Computer Science And Mathematics In Architecture And Civil Engineering Weimar Germany, Bauhaus University Weimar -pp 68-75 (2015)

20. A. Volkov, A. Sedov, P. Chelyshkov Usage of building information modelling for evaluation of energy efficiency / Applied Mechanics and Materials Vols. 409-410 -p 630 (2013)

21. P. Kagan, V. Kulikov Information modelling of urban planning development / Applied Mechanics and Materials Vols. 409-410 -p 951 (2013) 